\title{
System Design Analysis of a 0.22-THz Sheet-Beam Traveling-Wave Tube Amplifier
}

\author{
Young-Min Shin, Anisullah Baig, Larry R. Barnett, Wen-Ching Tsai, and Neville C. Luhmann, Jr., Fellow, IEEE
}

\begin{abstract}
The primary constituents of a 0.22 -terahertz (THz) sheet-beam traveling-wave tube (TWT) amplifier, composed of a staggered double grating array waveguide, have been designed for broadband $\mathrm{THz}$ operation $(\sim 30 \%)$ using the fundamental passband (TE-mode). Currently, we are looking into the possibility of a pulsed low-duty test of this device as a proof of principle (POP) and have been making efforts to construct the system. The optimally designed input coupler has $\leq 1 \mathrm{~dB}$ insertion loss at $0.22 \mathrm{THz}$ with 75 GHz (34\%) 1-dB matching bandwidths. A thin mica RF window provides a coupling bandwidth spanning multiple octaves. The collector is designed to have a jog for collecting the spent beam along the RF path coupled to the output RF window. Computer simulations show that the collector hybridized with a WR-4 window has $\sim 60 \mathrm{GHz}$ matching bandwidth with $\sim-0.5 \mathrm{~dB}$ insertion loss at 0.22 THz. The hybrid periodic permanent-magnet design combined with the quadrupole magnet (PPM-QM), intended for low-duty pulse operation in a proof-of-concept experiment, allows the elliptical sheet beam from an existing gun (25:1 aspect ratio) to unoptimized gun to have $73 \%$ beam transmission. The POP pulsed test is designed to be matched to our existing system, which will thereby tolerate beam transmission. However, a proper gun for the sheet-beam tunnel of the designed circuit will provide much better transmission. In our prior works, we successfully proved at W-band that the magnet design provided $>99 \%$ beam transmission of a 10:1 aspect ratio sheet beam. Most of the TWT circuit components have been designed, and currently, a full simulation modeling effort is being conducted.
\end{abstract}

Index Terms-Collector, coupler, sheet beam, terahertz (THz), traveling-wave tube (TWT), window.

\section{INTRODUCTION}

$\mathbf{T}$ $\mathrm{HE}$ recent rapid growth of terahertz (THz) technology [1], [2], frequently defined as frequencies from $100 \mathrm{GHz}$ to $30 \mathrm{THz}$ [3], [4], is having a major impact on advances in various modern scientific fields such as biomedical imaging, communication, and sensing as the electromagnetic waves have a variety

Manuscript received August 3, 2011; revised October 12, 2011; accepted October 14, 2011. Date of current version December 23, 2011. This work was supported by the High Frequency Integrated Vacuum Electronics (HiFIVE) Program of the Defense Advanced Research Projects Agency under Grant G8U543366. The review of this paper was arranged by Editor R. Carter.

Y.-M. Shin is with the Department of Physics, Northern Illinois University, DeKalb, IL 60115 USA, and also with the Accelerator Physics Center, Fermi National Accelerator Laboratory, Batavia, IL 60510 USA (e-mail: yshin@niu.edu; youngmin@fnal.gov).

A. Baig and L. R. Barnett are with the Department of Applied Science, University of California at Davis, Davis, CA 95616 USA (e-mail: abaig@ ucdavis.edu; mntntech@gmail.com).

W. C. Tsai and N. C. Luhmann, Jr. are with the Department of Electrical and Computer Engineering, University of California at Davis, Davis, CA 95616 USA (e-mail: wctsai@ucdavis.edu; ncluhmann@ucdavis.edu).

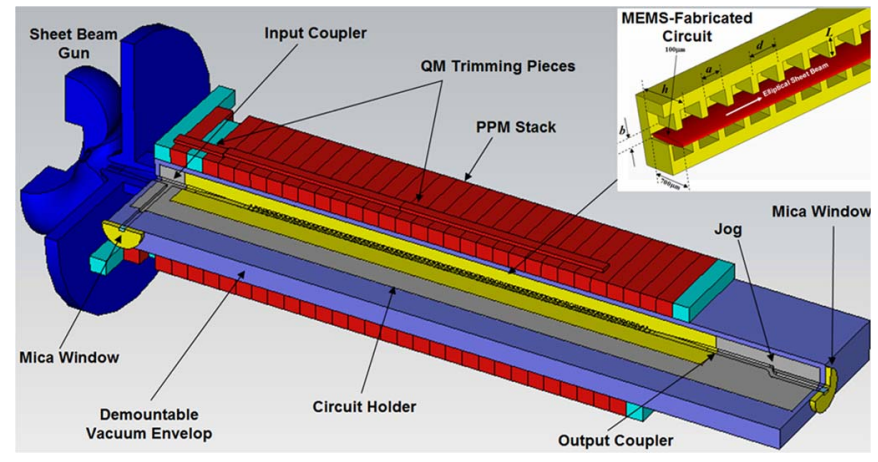

Fig. 1. Conceptual 3-D engineering drawing of the THz SBTWT amplifier, containing MEMS-fabricated staggered double-vane array structure (see the inset), coupler, window, collector, and magnet.

of physical properties merging electronics and photonics. In particular, $\mathrm{THz}$ waves are extremely useful for future communication applications demanding broad bandwidth and high signal-to-noise ratio as this frequency regime has large channel capacity and high data-conveyance rate. Relevant to this topic, a research program [5] on the development of a microfabricated $\mathrm{THz}$ sheet-beam traveling-wave tube (SBTWT) [6]-[8] amplifier was launched for THz-wave power module application [9]. In the course of pursuing this paper, we designed a novel SBTWT circuit structure composed of half-period-zigzagged double-vane arrays (TE-mode) [10]-[12], and our prior work on the numerical modeling analysis showed that the device will produce 150 - to $250-\mathrm{W}$ output power, corresponding to $\sim 3 \%-5.5 \%$ intrinsic electronic efficiency, overall efficiency value of $\sim 30 \%$ ( $100 \%$ beam transmission) with depressed collector (four-stage) operation, and $>1000 \mathrm{~W} \cdot \mathrm{GHz}$ powerbandwidth product, over $>25 \%$ bandwidth at the center frequency of $0.22 \mathrm{THz}$. The 3-D particle-in-cell (PIC) simulation modeling combined with large-signal 1-D analysis also corroborates stable operation of the designed circuit. Subsequently, we have investigated the system design of the TWT amplifier with a MEMS-fabricated interaction circuit for broadband component matching. Fig. 1 illustrates the conceptual engineering drawing of the SBTWT system intended for pulsed operation in a proofof-concept test, employing an existing sheet-beam gun [13], [14] used in the initial beam formation and propagation tests, input and output couplers, a collector, windows, and focusing magnet stack [15]. In the assembly concept, an SBTWT circuit, separately fabricated either by MEMS processes or nano-computer numerical control machining [16], [17], will be embedded in the demountable vacuum tube envelope. Here, it is noted that, although $0.22 \mathrm{THz}$ is at the lower end of the THz spectrum, in our prior works, $\sim 50 \mathrm{~nm}$ of surface roughness 
(which is smaller than the skin depth, i.e., $\sim 66 \mathrm{~nm}$, at $1 \mathrm{THz}$ on the SBTWT circuit) was achieved from the MEMS fabrications [18], and $>96 \%$ beam transmission of the required $7: 1$ aspect ratio sheet beam was demonstrated through our designed magnetic focusing structure by particle tracking simulations, which strongly support the viability of the SBTWT to full $\mathrm{THz}$ application. This paper discusses the designed models and numerical simulation analysis of the system components (i.e., coupler, window, collector, and focusing magnet) on the SBTWT output performance. Normally, severs are utilized in a high-gain TWT to stabilize device operation. PIC simulation showed that the 40-mm-long circuit has 34.8- to 37.4-dB gain so that the tube operation would become unstable with realistic input/output return loss of $\sim-10 \mathrm{~dB}$, resulting from intrinsic coupler and window mismatch, component misalignments, etc. It is, nevertheless, expected that increased ohmic loss owing to asperities, cracks, burrs, and so on will contribute to stabilizing the tube. Furthermore, as the proof-of-principle (POP) circuit may have lower beam transmission than predicted, the tube will be initially built as the simplest configuration without severs and attenuators. In the case that the high beam current causes an oscillation, an additional lossy metal coating on the circuit, which could be readily installed in the demountable tube system, would efficiently decrease signal growth rate, thereby stabilizing the system. Upper and/or lower cutoff oscillations can be also avoided by adjusting the circuit dimensions to disperse the passband, thereby segregating the beam line from the cutoffs. Separate from the system component design described in this paper, we performed a numerical analysis of the severed circuit model in PIC simulations, which will be published elsewhere.

\section{COUPLER}

Our interaction circuit design employs a 2-D planar structure with 7:1 aspect ratio, and it has beam focusing magnets in the narrow direction (minor axis) of the elliptical sheet beam. Consequently, the coupler structure is designed to be positioned along the wide direction (major axis) to avoid undesirable complexity of the coupler structure, which could possibly cause mismatching and attenuation. As shown in Fig. 2(a), the optimum input coupler design has a transition section of tapered vanes connected to the beam tunnel in the wide direction. Note that the $\mathrm{TE}_{10}$-mode oversized waveguide $\left(1220 \times 770 \mu \mathrm{m}^{2}\right)$ is embedded just after the coupler waveguide to maximize power mismatching toward the direction of the electron gun. In the time-transient simulation in Fig. 2(b), the frequency of the driving signal, fed through port 1 , is swept from 0.19 to $0.3 \mathrm{THz}$ and the transmitted power is measured at port 2 toward the circuit section and port 3 toward the gun section. In Fig. 3, the coupler is shown to have an $S_{21}$ of only $\sim-0.7 \mathrm{~dB}$, whereas $S_{31}$ is about $-14 \mathrm{~dB}$ at $0.22 \mathrm{THz}$. In addition, the 1-dB bandwidths of the designed coupler are $\sim 75 \mathrm{GHz}$, which corresponds to $\sim 34 \%$, for the desired $0.22-\mathrm{THz}$ operating frequency. Note that the matching bandwidth is gradually decreased with the increase in the size of the oversized waveguide and that trapped modes appear at $x=1200 \mu \mathrm{m}$. The oversized waveguide is embedded to induce
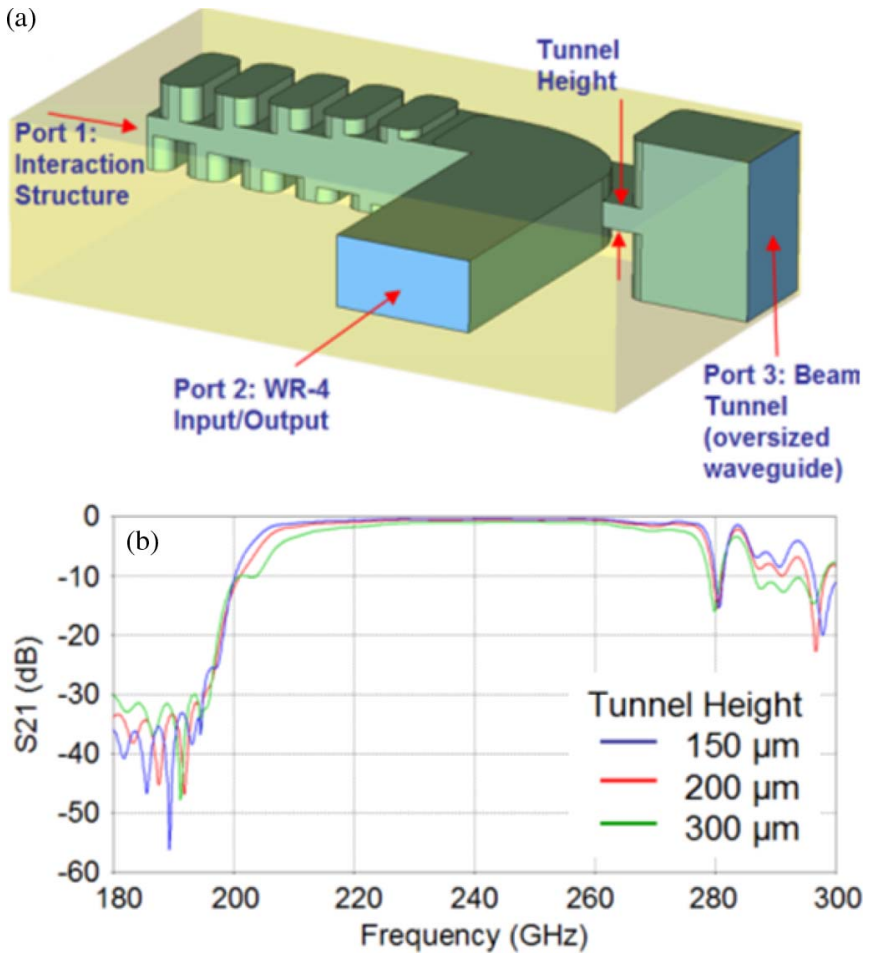

Fig. 2. (a) Designed L-bend coupler structure (oversized mismatching waveguide of rectangular type). (b) $S_{21}$ versus frequency graphs with respect to tunnel heights of the mismatching waveguide (toward port 2).

artificial mode conversion for outward broadband mismatching. In order to maintain $90^{\circ}$ polarization mismatching $\left(\mathrm{TE}_{10} \rightarrow\right.$ $\left.\mathrm{TE}_{01}\right)$ for broadband coupling, the oversized mismatching waveguides are designed to keep the height larger than the width. The trapped modes strongly resonate with the oversized waveguide, thereby leading to significant energy flow to port 3 (gun direction). As the height of the bottleneck beam tunnel between the oversized and L-bend waveguides is increased, the coupling bandwidth is reduced together with an increase in matching loss. The coupler models with $200-$ and $300-\mu \mathrm{m}$ thicknesses have 1-dB bandwidth and insertion loss $(0.22 \mathrm{THz})$ of $\sim 54 \mathrm{GHz}$ and $-1 \mathrm{~dB}(200 \mu \mathrm{m})$ and $\sim 45 \mathrm{GHz}$ and $-1.87 \mathrm{~dB}$ $(300 \mu \mathrm{m})$, respectively. Fig. 4 (see the inset) illustrates another coupler design modified with the cylindrical waveguide, which plays a role in increasing the mismatch toward the gun. This type of coupler provides a coupling bandwidth of $\sim 83 \mathrm{GHz}$ $(=\sim 38 \%)$ and matching loss of $\sim-0.8 \mathrm{~dB}$ at $0.22 \mathrm{THz}$, as shown in Fig. 4(a). Although the cylindrical-waveguide-type coupler has better matching characteristics, the rectangular one appears to be more compatible with high-frequency applications in the microfabrication aspect. The parametric simulations show that the coupler has robust coupling characteristics with respect to dimensional deviations. In Fig. 4(b), the coupling bandwidth remains relatively constant with respect to variation of the length of the tapered waveguide $x(1-5 \mathrm{~mm})$ having $\lesssim-1 \mathrm{~dB}$ insertion loss $(0.22 \mathrm{THz})$. Therefore, the bandwidth of the designed coupler is sufficiently broad to fully cover the intrinsic passband of the interaction circuit. It should be noted that this broadband matching coupler is designed with simple geometry, and it is compatible both with the hybrid periodic permanent-magnet design combined with the quadrupole 

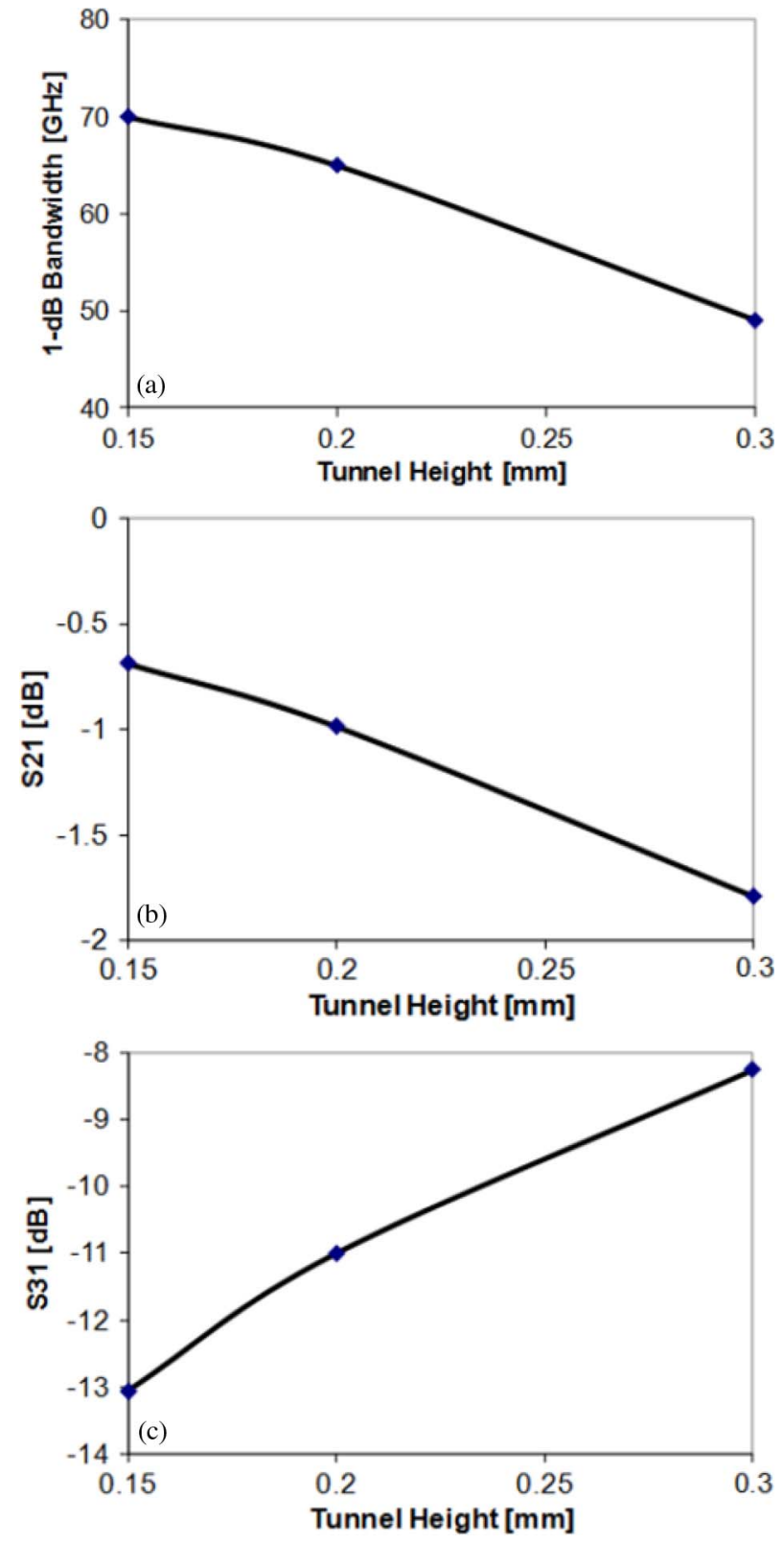

Fig. 3. Parametric analysis of the coupler (see Fig. 2). (a) Bandwidth of $1 \mathrm{~dB}$ and coupling losses toward (b) port 2 and (c) port 3 versus the height of the bottleneck beam tunnel.

magnet (PPM-QM) and 2-D microfabrication. The identical coupler design can be also considered for the output coupling and will be utilized in the continuous wave version.

\section{RF WINDOW}

A window is one of the most integral components in vacuum electron devices in which it couples RF energy in or out of the devices while preserving the vacuum state inside the tubes. A poorly designed window could lead to huge signal mismatches, thereby resulting in unacceptable loss in RF output power and matching bandwidth or parasitic oscillations. Since a staggered double grating array slow-wave circuit has a broadband operation range ( $\sim 30 \%)$, a pillbox mica window was investigated for both the designed input and output couplers due to its octave
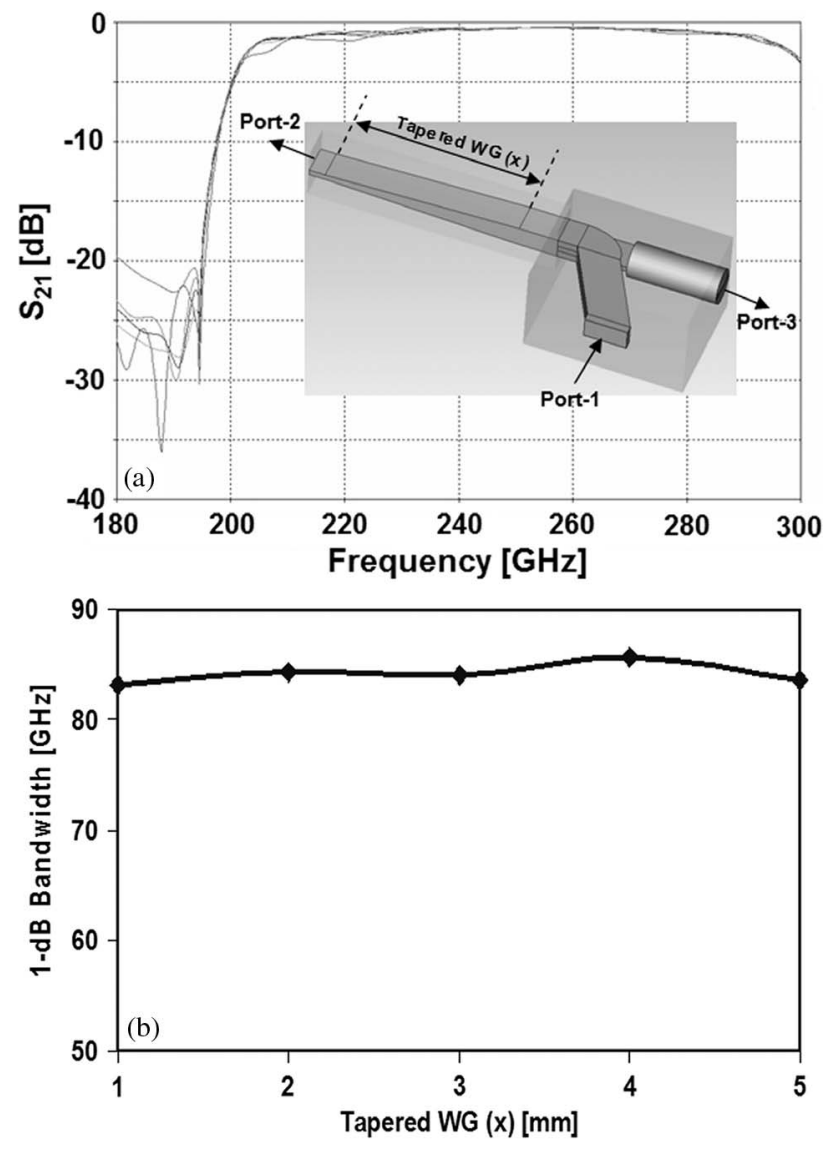

Fig. 4. Tapered straight waveguide coupler with mismatching load of cylindrical type (see the inset). (a) $S_{21}$ versus frequency graph with respect to the tapered section lengths. (b) Bandwidth of $1 \mathrm{~dB}$ versus tapered waveguide length.

bandwidth coverage. As a preliminary test, we modeled the rectangular window (WR-4) with $90^{\circ}$ sharp edges at the corners as their cutoff frequencies are well below the lower cutoff of the circuit passband. In Fig. 5(a), the window is composed of $\varepsilon_{r}=$ 4.5 lossy mica with $2.5-\mu \mathrm{m}$ thickness (radius $=2.5 \mathrm{~mm}$ ). A mica (monolayered crystal structure), a well-known RF window material, has sufficiently high mechanical strength to sustain high vacuum pressure. We successfully machined the mica to be the circular window without a crack using high-precision machining. In Fig. 5(b), the transient solver simulation analysis resulted in excellent matching with this WR-4 window, which has $S_{21} \cong 0 \mathrm{~dB}$ at $0.22 \mathrm{THz}$ with $0.8-\mathrm{THz} 1-\mathrm{dB}$ bandwidth $(\sim 360 \%)$. The designed mica window was machined to be 2.5- $\mu \mathrm{m}$ thick and attached to the WR-4 flange by Au-wire sealing for signal-coupling test. In Fig. 5(a), experimental results of $S_{21}=\sim 0 \mathrm{~dB}$ and $S_{11}<-10 \mathrm{~dB}$ are well matched with simulation data over the operational spectrum of 0.18 $0.265 \mathrm{THz}$. Currently, we are looking into the RF characteristics of thicker window structures, which may afford increased mechanical strength.

In addition, in order to prevent excitation of a higher order radial mode, if any, in the cylindrical mica window, as shown in Fig. 5(d), we designed a complementary window structure using a choke flange with the two concentric circular grooves to provide tight contact between the window and the waveguide. 

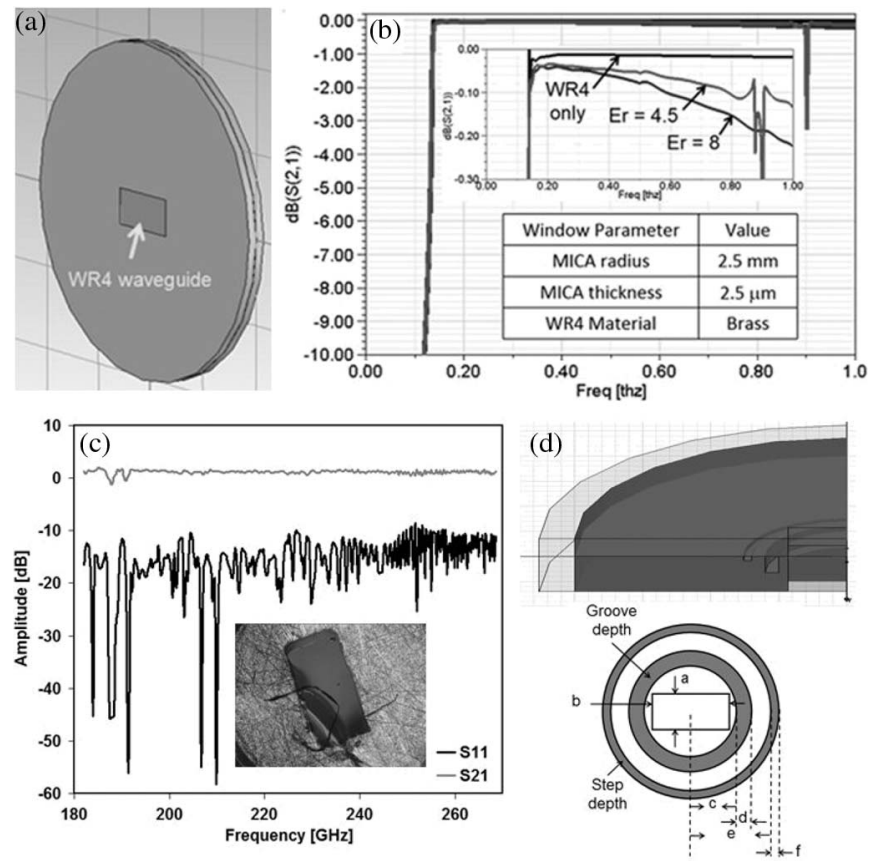

Fig. 5. Pillbox mica window of multiple octave bandwidths. (a) Drawing of the WR-4 window. (b) HFSS simulation result ( $S_{21}$ versus frequency). (c) Experimental RF test result ( $S_{11}$ and $S_{21}$ versus frequency). (d) RF window with a choke flange.

The choke flange window provides flat matching band response by enhancing longitudinal mode coupling with weakening mode coupling in the radial direction. The distance between the inner groove and the waveguide wall proportional to a quarter wavelength provides the optimum matching condition in which it cancels out the reflections and suppresses the radial coupled modes. The window coupling of a quarter-wavelength-deep inner groove appears to be quite robust even with respect to dimensional variations of $\pm 0.001 \mathrm{in}$. The latest design excludes the outer groove of the O-ring gasket as it hardly affects matching characteristics. The choke flange window would have the highest mechanical and thermal strength with the zero step. However, we are still investigating the optimum design with broad matching bandwidth and physical strength.

\section{Window-Type Collector}

Initial POP tests will be conducted under low-duty pulsed operation and there will thus only be a small amount of average spent beam power deposited on the collector, which thereby allows the collector to be combined with an output coupler as a single body. In the design, after passing the focusing B-field area, the spent beam is intentionally dumped to the collector wall due to intrinsic space-charge beam spreading, whereas the amplified RF signal is coupled out through the transition component from the tapered waveguide to the RF window. Fig. 6 depicts the designed output coupling collector structure comprising the tapered circuit transition part, tapered waveguide, and RF window. The level of beam current impinging upon the RF window was investigated as a function of the window position. A tapered waveguide length of $>44 \mathrm{~mm}$ is required for the electron interception to the window to vanish, which is
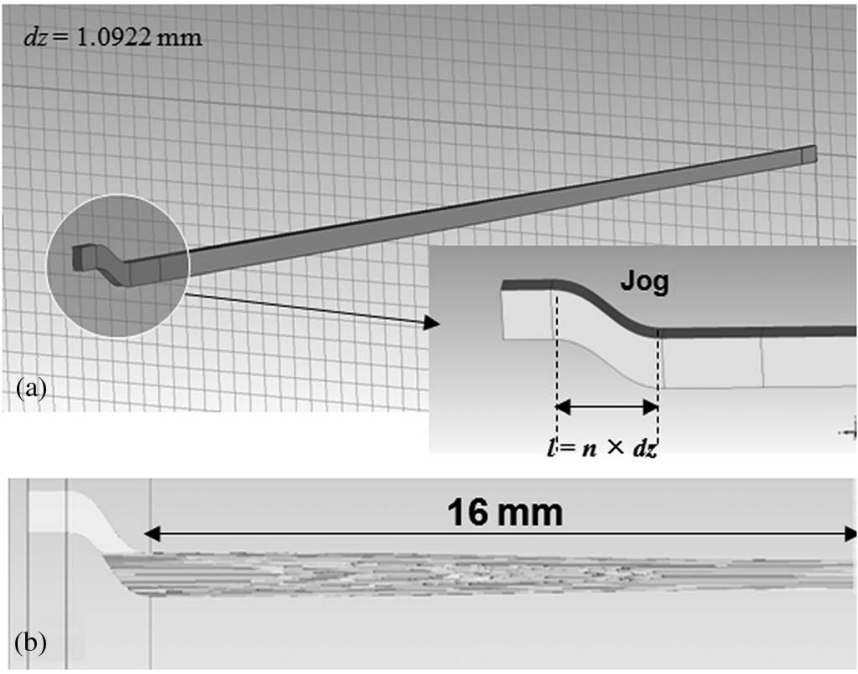

Fig. 6. (a) Conceptual 3-D drawing of the designed output coupler (with jog) (b) Beam trajectory plot (CST particle studio).
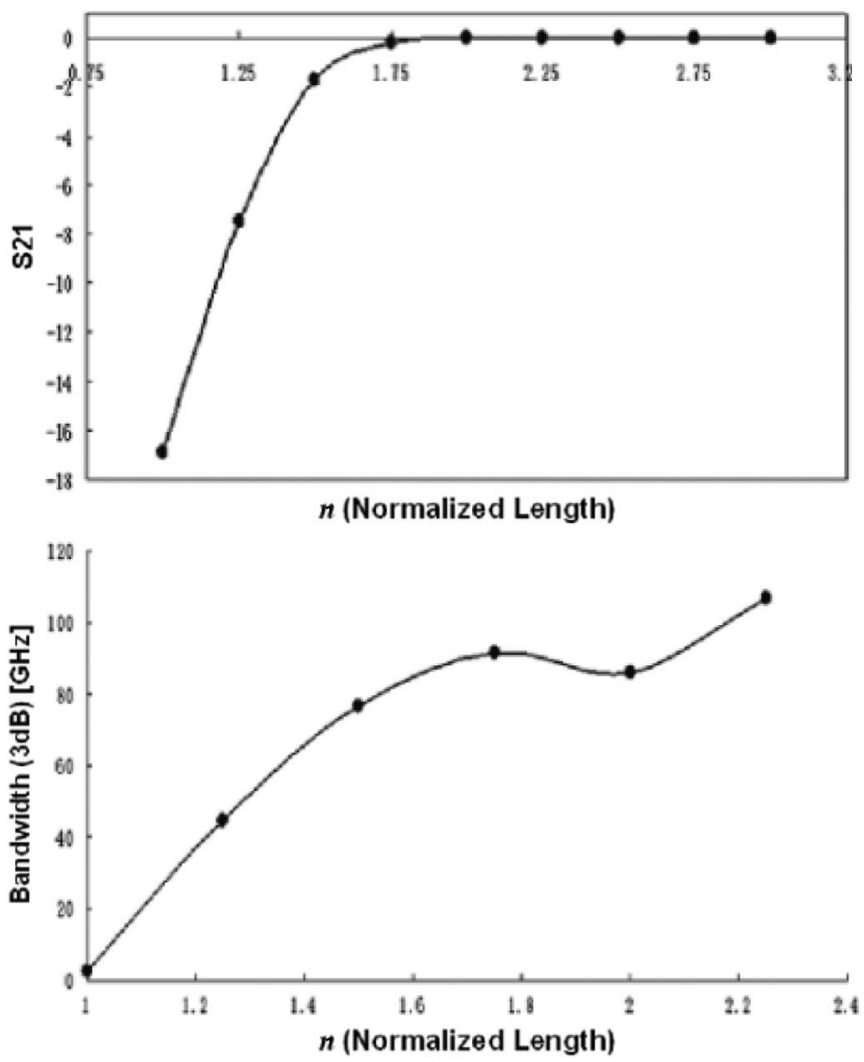

Fig. 7. Normalized tapered section length (unit: $n$ ) of the designed output coupler versus (a) $S_{21}(0.22 \mathrm{THz})$ and (b) 3-dB bandwidth.

sufficiently long in order to result in an unacceptable amount of attenuation to the output coupler. Therefore, the structure was redesigned with a minor modification to minimize the length of the transition part. In the second design, the window position is jogged off the beam trajectory in the transverse direction with the off shift as wide as the WR-4 waveguide, which will lead to the beam being fully intercepted at the corner of the waveguide bend. Fig. 7 shows the $S_{21}$ dependence upon frequency and the RF power density of the jogged collector model. The optimized model has $S_{21} \sim-0.5 \mathrm{~dB}$ at $220 \mathrm{GHz}$ 

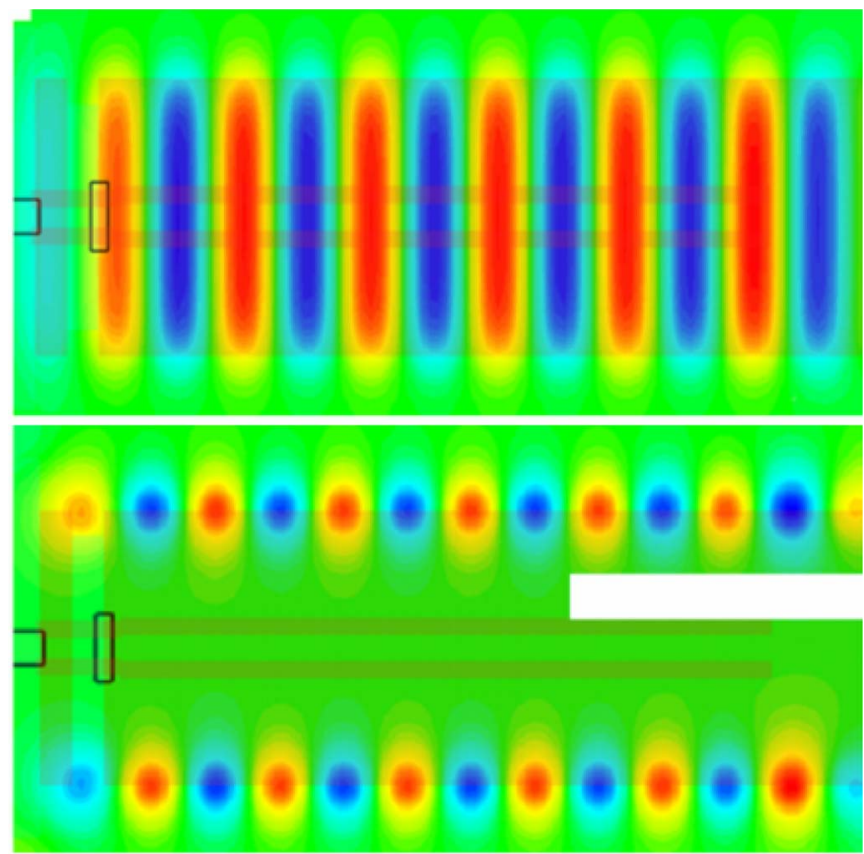

Fig. 8. Magnetic field distribution ( $B_{z}$ : left; $B_{x}$ : right) of the sheet-beam transport structure.

and 20\% 3-dB bandwidth. The particle tracking simulation with the designed model clearly proved that the window does not have any electron collisions in which no beam current is detected in the window port in the simulation.

\section{Hybrid Structure For Sheet-Beam Focusing}

As aforementioned, the planar circuit is designed for interaction with a sheet beam with dimensions of $700 \times 100 \mu \mathrm{m}^{2}(7: 1$ aspect ratio). In previous high-aspect-ratio SBTWT research [13], [14], an electron gun capable of producing an elliptical sheet beam of $15: 1$ aspect ratio was developed by Communications \& Power Industries (CPI), which produced an electron beam with 1200 (major) $\times 80$ (minor) $\mu \mathrm{m}^{2}$ cross-sectional area with $416-\mathrm{mA}$ emission current $\left(=552-\mathrm{A} / \mathrm{cm}^{2}\right.$ current density). To expedite experimental POP studies of the $0.22-\mathrm{THz}$ SBTWT, this gun (CPI Gun-\#2) will be used for initial POP SBTWT operation experiments as the beam current filtered by the $770 \times 150 \mu \mathrm{m}^{2}$ size beam tunnel contains sufficient beam power $(\sim 5 \mathrm{~kW})$ for coherent beam-wave interaction, and the dissipated energy of the electrons intercepted on the circuit wall will not be a problem in low-duty pulse operation. Fig. 1 shows the beam transport simulation model comprising the electron gun, the beam drift tube, and the focusing magnet structure. For beam focusing, we designed a hybrid magnetic lens structure consisting of the main periodic permanent-magnet (PPM) stack and the trimming quadrupole magnet $(\mathrm{QM})$ pieces. As shown in Fig. 8, the alternating magnetic field ( $B_{z}$ : top; $B_{x}$ : bottom) distribution of the PPM stack mainly provides the axial magnetic force to suppress the spreading beam with space-charge force in the $x$-axis, and the QM pieces play a role in precisely controlling the B-field components at the beam edge to focus the beam in the transverse direction ( $y$-axis). In the simulation model, the magnet pole pieces are designed to have a remanent
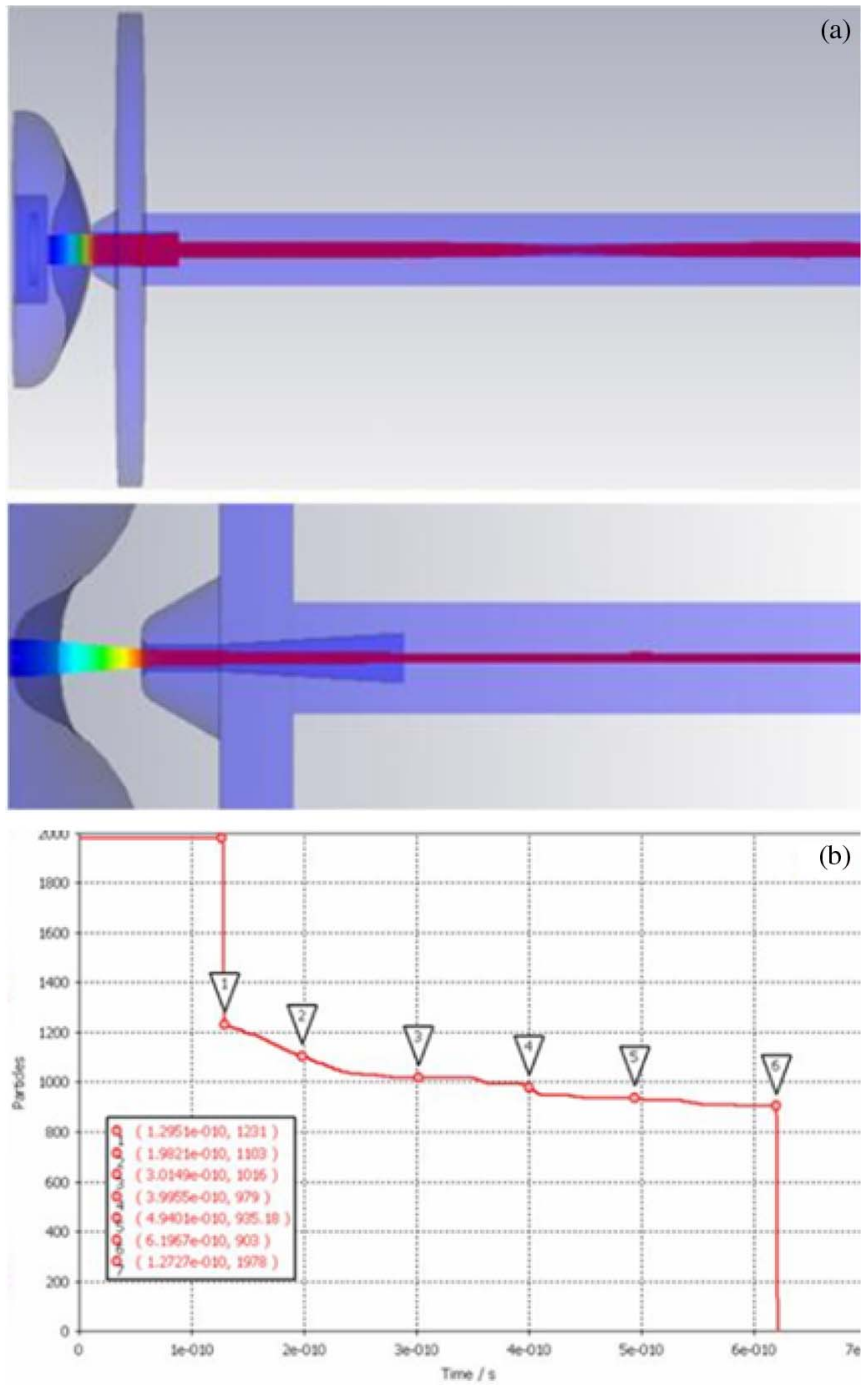

Fig. 9. (a) Beam trajectories of the elliptical sheet beam focused by the magnetic structure (top view: top; side view: bottom). (b) Axial distance versus beam transmission.

flux density $B_{r}$ of 1.4 T. Fig. 9 shows that the oversized beam emitted from the elliptical cathode and the intercepted beam current is electrostatically compressed down to $80 \mu \mathrm{m}$ (minor axis) in thickness and focused through the beam tunnel by the magnetic focusing field (see Fig. 8) of the periodic magnet structure. The beam shape maintains a width of $1200 \mu \mathrm{m}$ (major axis) and impinges upon the circuit entrance, whereas the remaining $62.23 \%(=259 \mathrm{~mA})$ of the beam propagates in the beam tunnel. As the beam is much thinner and wider than the beam tunnel, beam interception only occurs at both sides of the tunnel in the wider direction. It turned out with the particle tracking simulation that there is $73.36 \%$ beam transmission ( $\cong 190 \mathrm{~mA}$ ) along the 4-cm-long drift tube. Note that, according to the PIC simulation analysis, the circuit produces more than $150 \mathrm{~W}$ with $250 \mathrm{~mA}$; hence, $190 \mathrm{~mA}$ is a sufficiently high beam current to produce an appreciable amount of radiation power, i.e., $100-130 \mathrm{~W}$, beyond the minimum criterion for practical application $(\sim 50 \mathrm{~W})$. Fig. 9 (b) displays the beam interception along the axial distance, indicating that the beam gradually diverges at the end of the circuit. It is anticipated that 
beam transmission can be improved by further optimization of magnet design parameters, particularly the gap spacing between trimming QM pieces. In prior work, we successfully obtained $>99 \%$ RF-induced beam transmission of a 10:1 aspect ratio sheet beam (58 kV and $2.48 \mathrm{~A}$ ) using the same PPM-QM design in a W-band sheet-beam klystron. Thus, we expect that the current PPM-QM design for this SBTWT application will produce much higher beam transmission with a properly designed electron gun for the required $7: 1$ aspect ratio sheet beam. Currently, the device component design is being fully optimized by a more systematic investigation.

\section{CONCLUSION}

The SBTWT components, including input/output couplers, RF window, collector, and magnet structure, have been fully designed, and currently, their dimensional parameters are being optimized. Commonly, a high-gain TWT amplifier necessitates a sever to eliminate backward waves for stable operation. However, as aforementioned, intrinsic ohmic loss of the machined circuit due to various imperfections such as rough surfaces, defects, burrs, and so on may give rise to a sufficient amount of signal attenuation to stabilize tube operation without a sever. The first tube for the proof-of-concept pulse test, thus, will not include a sever, whereas we already studied the effects of a sever on the device performance, which will be presented through a separate publication. This test will corroborate the viability of the ultrabroadband SBTWT scheme for THz application in which we already achieved $\sim 50 \mathrm{~nm}$ of surface roughness by MEMS fabrication, which is smaller than the skin depth, i.e., $\sim 66 \mathrm{~nm}$, at $1 \mathrm{THz}$. Although some technical challenges still remain, the accomplishments on this paper will pave the way for achieving successful development of a Wattlevel $\mathrm{THz}$ radiation source.

\section{REFERENCES}

[1] P. H. Siegel, "Terahertz technology," IEEE Trans. Microw. Theory, vol. 50, no. 3, pp. 910-928, Mar. 2002.

[2] J. H. Booske, "Plasma physics and related challenges of millimeterwave-to-terahertz and high power microwave generation," Phys. Plasmas, vol. 15, no. 5, p. 055502, May 2008.

[3] M. Tonouchi, "Cutting-edge terahertz technology," Nat. Photon., vol. 1, no. 2, pp. 97-105, Feb. 2007.

[4] H. B. Wallace, "Analysis of RF imaging applications at frequencies over 100 GHz," Appl. Opt., vol. 49, no. 19, pp. E38-E47, Jul. 2010.

[5] [Online]. Available: http://www.darpa.mil/mto/programs/hifive/index. html

[6] R. L. Ives, "Microfabrication of high-frequency vacuum electron devices," IEEE Trans. Plasma Sci., vol. 32, no. 3, pp. 1277-1291, Jun. 2004.

[7] H. Zhang, J. Wang, C. Tong, X. Li, and G. Wang, "Numerical studies of powerful terahertz pulse generation from a super-radiant surface wave oscillator," Phys. Plasmas, vol. 16, no. 12, p. 123 104, Dec. 2009.

[8] G. S. Nusinovich, S. J. Cooke, M. Botton, and B. Levush, "Wave coupling in sheet- and multiple-beam traveling-wave tubes," Phys. Plasmas, vol. 16, no. 6, p. 063 102, Jun. 2009.

[9] [Online]. Available: http://www.darpa.mil/MTO/Solicitations/baa07-49/ HiFIVE_Overview.pdf

[10] Y.-M. Shin and L. R. Barnett, "Intense wideband terahertz amplification using phase shifted periodic electron-plasmon coupling," Appl. Phys. Lett., vol. 92, no. 9, p. 091501, Mar. 2008.

[11] Y.-M. Shin, L. R. Barnett, and N. C. Luhmann, Jr., "Strongly confined plasmonic wave propagation through an ultrawideband staggered double grating waveguide," Appl. Phys. Lett., vol. 93, no. 22, p. 221504, Dec. 2008.
[12] Y.-M. Shin, L. R. Barnett, and N. C. Luhmann, Jr., "Phase-shifted traveling-wave-tube circuit for ultrawideband high-power submillimeterwave generation," IEEE Trans. Electron. Devices, vol. 56, no. 5, pp. $706-$ 712, May 2009.

[13] D. Gamzina, A. G. Spear, L. R. Barnett, and N. C. Luhmann, Jr., "THz sheet beam gun analyzer," in Proc. IEEE IVEC, 2010, pp. 99-100.

[14] J. E. Atkinson, D. D. Gajaria, T. J. Grant, T. Kimura, B. C. Stockwell, M. Field, R. J. Borwick, B. Brar, and J. A. Pasour, "A high aspect ratio, high current density sheet beam electron gun," in Proc. IEEE IVEC, 2010, pp. $97-98$.

[15] J.-X. Wang, L. R. Barnett, S. Humphries, N. C. Luhmann, Jr., and Y.-M. Shin, "Electron beam transport analysis of W-band sheet beam Klystron (WSBK)," Phys. Plasmas, vol. 17, no. 4, p. 043111, Apr. 2010.

[16] Y.-M. Shin, A. Baig, A. Spear, J. Zhao, D. Gamzina, C. W. Domier, and N. C. Luhmann, Jr., "MEMS fabrications of broadband epsilon negative (ENG) metamaterial electronic circuit for $0.22 \mathrm{THz}$ sheet beam TWT application," in Proc. 35th Int. Conf. IRMMW-THz, 2010, pp. 1-2.

[17] R. A. Barchfeld, D. Gamzina, Y.-M. Shin, J.-S. Kim, L. R. Barnett, and N. C. Luhmann, "Microfabrication of terahertz vacuum electron devices," in Proc. 26th Amer. Soc. Precision Eng., 2011. in press.

[18] Y.-M. Shin, L. R. Barnett, D. Gamzina, N. C. Luhmann, Jr., M. Field, and R. Borwick, "Terahertz vacuum electronic circuits fabricated by UV lithographic molding and deep reactive ion etching," Appl. Phys. Lett., vol. 95, no. 18, p. 181505, Nov. 2009.

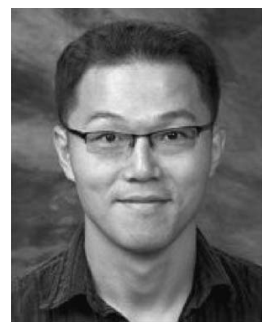

Young-Min Shin received the B.S. degree from Chung-Ang University, Seoul, Korea, in 1999 and the Ph.D. degree in physics from Seoul National University, Seoul, Korea, in 2006.

From 2001 to 2003, he was a Visiting Researcher with the Klystron/Microwave Department, Stanford Linear Accelerator Center, Menlo Park, $\mathrm{CA}$. He has worked on various millimeter- and submillimeter-wave microvacuum electron devices, including traveling-wave tubes and klystrons. He was a Research Scientist with the Department of Applied Science, University of California, Davis. He is currently an Assistant Professor with the Department of Physics, Northern Illinois University, DeKalb, and an Associate Scientist with the Accelerator Physics Center, Fermi National Accelerator Laboratory, Batavia, IL. Recently, his research interest has been mainly focused on MEMS fabrication/integration of novel terahertz vacuum sources combined with surface plasmonic metamaterials in near-field optics.

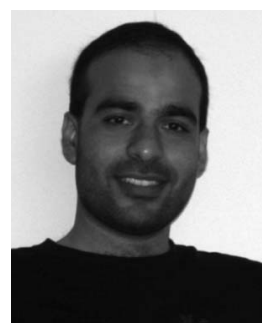

Anisullah Baig received the M.Sc. and M.Phil. degrees (summa cum laude) in physics from Quaid-i-Azam University, Islamabad, Pakistan. He is currently working toward the Ph.D. degree with the Millimeter Wave Technology Group, Department of Engineering and Applied Science, University of California, Davis, Davis.

From August 2007 to March 2008, He was a Synchrotron Light for Experimental Science and Applications in Middle East Training Fellow in the field of "accelerators physics and applications" with the Synchrotron Radiation Facility, Daresbury Laboratory, Warrington, U.K., with the project being developed under the auspices of UNESCO. His current research interests include the development of millimeter-wave band/terahertz sheet-beam vacuum electron devices (TWT, Klystron), MEMS fabrication, computational modeling/simulation, and radio-frequency measurements.

Mr. Baig was the recipient of an outstanding poster presentation award and cash prize for his M.Phil. research from the Pakistan Physical Society, the prestigious "Fulbright Scholarship" for Ph.D studies in the U.S., and the "2010 Richard Snavely Memorial Award" for excellence in academics and research from the Department of Engineering and Applied Science, University of California, Davis 


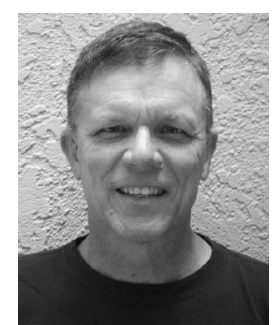

Larry R. Barnett received the B.S.E.E. degree from Tennessee Technological University, Cookeville, in 1972, the M.S.E.E. degree from The University of Tennessee Space Institute, Tullahoma, in 1975, and the $\mathrm{Ph} . \mathrm{D}$. degree with major in electrical engineering and minor in physics from The University of Tennessee, Knoxville, in 1978.

He began his professional association at the Naval Research Laboratory, Washington, DC, in the Plasma Physics Division, first as a National Research Council Associate and then as a Research Physicist until 1983. From 1983 to 1990, he was a Research Assistant Professor and then a Research Associate Professor with the Electrical Engineering Department, University of Utah, Salt Lake City, working on the submillimeter-wave backward-wave oscillators and harmonic gyrotron. In 1989, he began independent research, development, and consulting DBA Mountain Technology. He was located in Colorado from 1989 to 1994, and in 1995, he relocated to Normandy, TN. He has continuing collaboration with the Department of
Physics, National Tsing Hua University, Hsinchu, Taiwan, on the gyrotraveling-wave tube (TWT), gyrotron, and RF heating projects. He is currently collaborating with the Department of Applied Science, University of California, Davis, on high-power millimeter-wave devices, including the gyroTWT, gyrotron, sheet-beam klystron, and sheet-beam TWT. He is currently working on a Naval Sea Systems Command (NAVSEA) Small Business Innovation Research (SBIR) program to develop a 95-GHz gyrotron using a normal magnet.

Wen-Ching Tsai, photograph and biography not available at the time of publication.

Neville C. Luhmann, Jr. (SM'95-F'02), photograph and biography not available at the time of publication. 